\title{
Pharmacokinetic Concentration Method
}

National Cancer Institute

\section{Source}

National Cancer Institute. Pharmacokinetic Concentration Method. NCI Thesaurus. Code C87960.

The technique used to administer an examination or assessment of pharmacokinetic concentration. 